
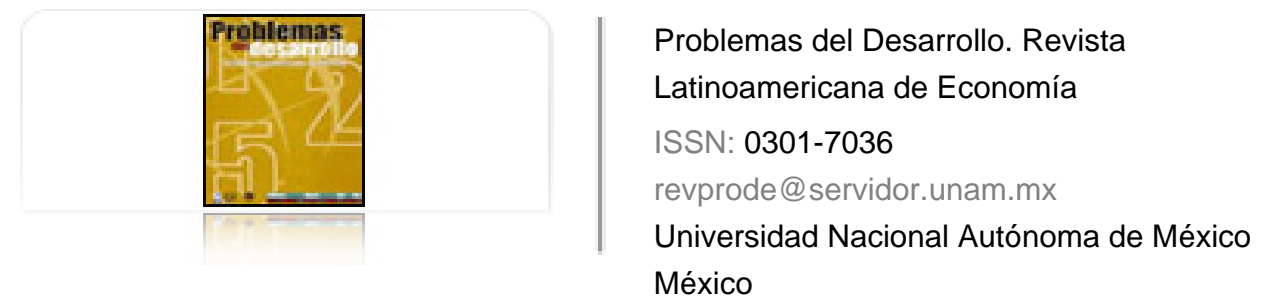

Becerril, Javier; Ortiz, Rafael; Albornoz, Lilian

Maquiladoras e ingreso de los hogares en Yucatán

Problemas del Desarrollo. Revista Latinoamericana de Economía, vol. 43, núm. 171, octubre-

diciembre, 2012, pp. 135-160

Universidad Nacional Autónoma de México

Distrito Federal, México

Disponible en: http://www.redalyc.org/articulo.oa?id=11823951007

- Cómo citar el artículo

- Número completo

- Más información del artículo

Página de la revista en redalyc.org

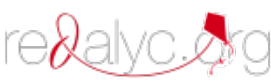

Sistema de Información Científica

Red de Revistas Científicas de América Latina, el Caribe, España y Portugal Proyecto académico sin fines de lucro, desarrollado bajo la iniciativa de acceso abierto 


\title{
Maquiladoras E ingreso de los hogares en YuCatán ${ }^{1}$
}

\author{
Javier Becerril, ${ }^{*}$ Rafael Ortiz ${ }^{* *}$ y Lilian Albornoz ${ }^{* * *}$
}

Fecha de recepción: 25 de septiembre del 2011. Fecha de aceptación: 2 de mayo del 2012.

\section{RESUMEN}

Este estudio examina los efectos de la industria maquiladora de exportación (IME) sobre el ingreso neto completo anual de los hogares rurales en la antigua región henequenera de Yucatán. Éste emplea el enfoque Propensity Score Matching y datos de corte transversal de 1123 habitantes que conforman 247 hogares de cuatro municipios: Cuzamá, Homún, Acanceh y Huhí. Los hallazgos revelan un impacto robusto positivo y significativo en el ingreso total anual de los habitantes que laboran para la IME establecida en la región rural de Yucatán. Específicamente existe una diferencia positiva que va de $\$ 8,419$ a $\$ 9,449$ pesos mexicanos anuales entre los habitantes que laboran en una maquiladora y los que laboran en cualquier otro tipo de actividad dentro o fuera del territorio.

Palabras clave: Maya, rural, Yucatán, maquila, ingreso, Propensity Score-Matching.

\section{Maquiladora Factories and Household income in Yucatan}

\begin{abstract}
This study examines the effect of the maquiladora export industry (MEI) on total net annual household income for rural zones of the old Henequenera region of Yucatán. This analysis uses the Propensity Score Matching approach and cross-sectional data from 1,123 inhabitants that make up 247 households in four municipalities: Cuzamá, Homún, Acanceh and Huhí. The findings reveal a strong and significant positive impact on the total annual income of inhabitants working for the MEI in the rural region of Yucatan. Specifically, there is a positive difference that ranges from $\$ 8,419$ to $\$ 9,449$ Mexican pesos annually between inhabitants that work for a maquiladora factory and those that work in any other type of activity within or outside of the territory.
\end{abstract}

Key Words: maya, rural, Yucatán, maquila, income, Propensity Score-Matching.

\footnotetext{
1 Los autores agradecen a todos los habitantes que contribuyeron al responder las encuestas aplicadas a los hogares; a todos los encuestadores: Carolina Solís Flores, Carlos Eduardo Robleda Chávez, Jesús Filemón Hernández Santoyo y Omar Antonio Burgos Maldonado que participaron en el proyecto de investigación, etapas 2A y 2B. A los patrocinadores del proyecto: PRECesAm, del Centro de Estudios Económicos de El Colegio de México, A. C.; al RIMISP-Centro Latinoamericano para el Desarrollo Rural; al Consejo Nacional de Ciencia y Tecnología (CONACYT); y a la Facultad de Economía de la Universidad Autónoma de Yucatán (UADY). Cualquier error u omisión en el estudio es responsabilidad de los autores.

* Profesor-Investigador de la Facultad de Economía, Universidad Autónoma de Yucatán. Correo electrónico: javier.becerril@uady.mx. Colaborador del Programa de Estudios del Cambio Económico y la Sustentabilidad del Agro Mexicano (precesam), del Centro de Estudios Económicos de El Colegio de México.

** Profesor-Investigador de la Facultad de Economía, Universidad Autónoma de Yucatán. Correo electrónico: rafael.ortiz@uady.mx

*** Profesora-Investigadora de la Facultad de Economía, Universidad Autónoma de Yucatán. Correo electrónico: lilian.albornoz@uady.mx
} 


\section{DES USINES ET LE REVENU DES FOYERS À YUCATÁN \\ Résumé}

Cette étude examine les effets de la sous-traitance industrielle pour l'exportation (IME, Industria Maquiladora de Exportación) sur le revenu net complet annuel des foyers ruraux au Yucatán, région anciennement vouée à la production de henequen. Elle adopte la démarche du Propensity Score Matching et se base sur des données de coupe transversale de 1123 habitants constituant 247 foyers de quatre municipalités : Cuzama, Homun, Acanceh et Huhi. Les résultats montrent une forte répercussion positive et significative sur le revenu total annuel des habitants qui travaillent pour l'ıme établie dans la région rurale du Yucatan. Il existe notamment une différence positive variant de 8.419 à 9.449 pesos mexicains annuels entre les habitants qui travaillent dans une usine d'assemblage et ceux qui travaillent dans un autre type d'activité à l'intérieur ou à l'extérieur du territoire.

Mots clés: maya, rural, Yucatán, sous-traitance industrielle, revenu, Propensity ScoreMatching.

\section{MAQUILADORAS E RENDA DOS LARES EM YUCATÁN. \\ Resumo}

Esse estudo examina os efeitos da indústria maquiladora de exportação (IME) sobre a renda liquida completa anual dos domicílios rurais da antiga regiáo produtora de fibra de henequén em Yucatán. Este emprega o enfoque Propensity Score Matching e dados de corte transversal de 1123 habitantes que conformam 247 domicílios de quatro municípios: Cuzamá, Homún, Acanceh e Huhí. Os resultados encontrados revelam um impacto robusto positivo e significativo na renda total anual dos habitantes que trabalham para a IME estabelecida na zona rural de Yucatán. De maneira especifica, existe uma diferença positiva que vai de $\$ 8.419$ a $\$ 9.449$ pesos mexicanos anuais entre os habitantes que trabalham numa maquiladora e os que trabalham em qualquer outro tipo de atividade dentro ou fora do território.

Palavras-chave: maia, rural, Yucatán, maquila, renda, Propensity Score-Matching.

\section{客户工业对尤卡坦州四个自治市家庭收入的影响}

摘要

本文研究了客户出口工业对尤卡坦州Henequenera农村地区家 庭收入的年度影响。本文使用了“倾向度匹配”方法，并从

Cuzam á 、Hom ún、Acanceh和Huh í 四个自治市抽样了由 247 个家 庭、1123居民构成的跨行业数据。研究发现, 若尤卡坦州农村地区家庭成 员在客户出口工业部门工作, 则对该家庭的年度收入有着显著影响。具体 而言, 对在客户工业部门工作的居民收入与在其它部门工作的居民收入相 比较, 这种收入年度影响差异是前者要高于后者8419 9449. 墨西哥比索。 关键词：玛雅 农村尤卡坦 客户工业收入倾向度匹配 


\section{INTRODUCCIÓN}

El compromiso con el desarrollo económico regional ha alentado a los gobiernos de los países a la instrumentación de políticas tendentes a promover el empleo, disminuir la pobreza y a dinamizar el mercado interno y externo. México no está exento de estos compromisos con el desarrollo económico regional; para ello ha impulsado múltiples mecanismos a diferentes escalas para promover la inversión interna y externa con miras a generar fuentes de empleo, mitigar la migración y el alivio de la pobreza. La industria maquiladora de exportación ${ }^{2}$ (IME) ha sido un mecanismo importante en la contribución al proceso de industrialización en México (Trívez, Reyes y Aliaga, 2007; Gómez, 2004). La IME no deja de ser uno de los sectores estratégicos sobre los que se encuentra apuntalada una considerable parte de la economía del país y de los estados.

Existe abundante literatura sobre el tema de la IME mexicana desde visiones diversas de la economía como ciencia social, ya que en muchas de estas investigaciones se describen aspectos históricos sobre el surgimiento de la industria maquiladora, las políticas públicas que la favorecieron, y su evolución y expansión de los estados fronterizos del norte de México hacia otras regiones y entidades federativas (Sklair, 1993; Wilson, 1992; Castilla y Torres, 2009; Warden, 2000). Algunos son estudios sobre casos específicos como los pagos otorgados y vida social de trabajadores(as) de maquiladoras (Carrillo y Hualde, 1997; Gómez, 2004; García et al., 2009). Otros se enfocan al género, la sociología, y disciplinas afines (Castilla y Torres, 2009; Taylor, 2010; Veloz, 2010); y aunque muchos de los estudios sobre el tema han hecho importantes contribuciones desde diferentes perspectivas, aún existe cierta ausencia en la literatura de trabajos de investigación que se enfoquen explícitamente al análisis del impacto económico, con excepción de Biles (2004) que evalúa cómo las maquiladoras impactan en el crecimiento económico en ciertas regiones de Yucatán por la distribución del ingreso a nivel macroeconómico.

2 De acuerdo con Gómez (2004) se entiende como industria maquiladora de exportación a una entidad que ensambla, manufactura, procesa o repara materiales temporalmente importados por el país receptor para su eventual reexportación o envío a su país de origen. 


\section{Origen y contexto de la industria maquiladora de exportación en Yucatán}

De acuerdo con Quintal (2010), desde finales del siglo XIx hasta la década de los años 1980 la región centro-sur de Yucatán formó parte de la zona cuya economía se basó en el cultivo del henequén, agave endémico de la península cuya fibra fue muy demandada en el mercado internacional hasta mediados del siglo $\mathrm{xx}$, cuando inició la producción mundial de sustitutos artificiales. Ante el desplome de la demanda de la fibra del henequén, en la segunda mitad de los años ochenta, y hasta finales de ese siglo la industria maquiladora se convirtió en la principal actividad económica de la región.

El análisis de la IME es importante desde que se estableció en el estado como generadora de empleo. De acuerdo con Castilla y Torres (2009) la presencia del capital extranjero de la IME en Yucatán se divide en dos etapas: i) la primera que va de 1984 a 1994 donde el capital extranjero se instaló en la capital yucateca, Mérida, en los corredores industriales; ii) la segunda que inicia a la mitad de 1990, al implementarse el programa Desarrollo Regional de la Zona Henequenera, dando inicio al establecimiento de la IME en municipios y localidades rurales y no rurales del interior del estado, coincidiendo a nivel nacional con la entrada en vigor del Tratado de Libre Comercio con América del Norte (TLCAN) en 1994. La ime cobró especial auge como resultado de la firma del TLCAN y las previsiones contenidas en dicho tratado, relativas al fomento de las maquilas, ampliando particularmente la posibilidad de las empresas manufactureras para operar bajo el esquema de maquila, y poder realizar importaciones temporales de todos aquellos insumos y producir para la exportación (Gómez, 2004). En los últimos ańos se ha detectado en México un desplazamiento de las maquilas más demandantes de mano de obra no calificada desde el norte y centro del país hacia estados ribereños de El Caribe, como Yucatán, donde los salarios son menores que en el resto de la República.

Adicionalmente, en 2006 el Programa de Fomento y Operación de la IME y el Programa de Importación Temporal para producir artículos de exportación (PITEX) se fusionan para dar lugar al Programa de Fomento a la Industria Manufacturera, Maquiladora y de Servicios de Exportación (IMMEx), que de acuerdo con los autores en 2009 había en México 6,694 empresas registradas en IMMEX, y para ese mismo año en Yucatán se reportaron 93 establecimientos manufactureros y maquiladores, que en conjunto generaron 18,858 empleos. De la industria IMmex establecida en Yucatán, el 85\% exporta a los Estados Unidos de América (EUA) (Secretaría de Fomento Económico, 2009).

Con estos antecedentes, la motivación de este estudio partió de los resultados del Programa Dinámicas Territoriales Rurales (DTR) Yucatán, realizado 
inicialmente en el año 2009. Dicho programa consistió en tres etapas 1A, 2A y 2B (Yúnez et al., 2009 y Yúnez et al., 2011). La etapa 1A descansó en el análisis de una tipología de territorios rurales basada en mejoras o detrimentos en el nivel de bienestar de los habitantes en el plano municipal para los años comprendidos entre 1990 y 2005. En aquel estudio los niveles de bienestar se consideraron: consumo per cápita, incidencia de la pobreza y coeficientes de Gini. Uno de los territorios que presentó una dinámica positiva y mejoras en los tres indicadores fue el conformado por los cuatro municipios Cuzamá, Homún, Acanceh y Huhí (CHAH en adelante) propuestos aquí para su estudio. Con los resultados de dicha investigación se sugirió una serie de territorios para ser estudiados a profundidad por el DTR etapa $2 \mathrm{~A}$ y $2 \mathrm{~B}$.

En las etapas 2A y 2B del programa DTR-Yucatán, Yúnez et al. (2011) analizan la dinámica del territorio CHAH para el periodo que va de $1990 \mathrm{a}$ 2010. Uno de los principales hallazgos es el cambio del predominio de las actividades eminentemente agrícolas hacia la diversificación de actividades económicas y de fuentes de ingreso de los habitantes del territorio. La transformación económica del CHAH se puede observar a partir del fin de la agroindustria henequenera paraestatal de principios de los ańos noventa, con el inicio de diversificación de actividades productivas generada por la relación entre factores exógenos y endógenos al territorio. Entre los primeros destacan las acciones gubernamentales a partir de la implementación del Programa de Reorientación de la Zona Henequenera y Desarrollo Integral de Yucatán en 1984, aunado con las fuentes de empleo promovidas por el crecimiento fuera del territorio y el establecimiento de la IME.

\section{Objetivos de análisis}

Este estudio analiza la importancia del ingreso total anual por individuo que trabaja para una maquiladora, versus otro tipo de empleo ofrecido en un territorio específico, con un enfoque cuantitativo riguroso y a un nivel de detalle territorial, de hogar e individuo (microeconómico) que no se había hecho anteriormente en la región.

El objetivo particular que sigue este artículo es efectuar una evaluación de impactos de la IME en un territorio conformado por cuatro municipios: Cuzamá, Homún, Acanceh y Huhí. Además tiene componentes académicos, pues ofrece una metodología alternativa para el análisis cuantitativo de evaluación de impactos económicos del sector maquilador de exportación establecido en Yucatán a mediados de 1990, y además es útil para orientar el diseño, 
implementación y evaluación de programas de política pública en México o Yucatán, con el fin de incentivar el desarrollo económico regional.

Concretamente a partir del establecimiento de la IME, conocer cómo los ingresos recibidos por los habitantes de la СНAH que se dividieron entre trabajadores de IME y no IME impactan en la economía del territorio. Para lograrlo se presentan los instrumentos de análisis, fuentes de información y análisis de información, y se termina con la discusión de los resultados obtenidos.

\section{INSTRUMENTO DE ANÁLISIS}

La relación básica que considera el estudio para examinar el impacto de la decisión de laborar para una IME, es el supuesto de bienestar que los individuos asumen medido a partir de su ingreso, como una función lineal de un vector de variables explicativas $\left(X_{i}\right)$ y una variable dicotómica de decisión $\left(R_{i}\right)$. La ecuación de la regresión lineal se puede especificar como:

$Y_{i}=X_{i}^{\prime} \beta+\delta R_{i}+u_{i}$

Donde $Y_{i}$ es el ingreso promedio de un individuo $i$; la literal $u_{i}$ es el término de error aleatorio con distribución normal y $R_{i}$ es la variable dicotómica 1 o 0 sobre la decisión de laborar o no en una IME; $R_{i}=1$ si el individuo labora para la IME y $R_{i}=0$ en cualquier otro tipo de empleo. El vector $X_{i}$ representa las características del individuo, su hogar y la localidad donde habita. La decisión de los individuos de trabajar o no trabajar en una IME es independiente del perfil de los individuos o de sus hogares, porque la decisión de un individuo de laborar para una IME está basada en sus propias decisiones, es decir, no es una decisión aleatoria.

Suponiendo que los individuos asumen un riesgo-neutral en la decisión de laborar para la IME, entonces la función sobre la decisión de emplearse en una IME se puede expresar como:

$R_{i}^{*}=X_{i}^{\prime} \gamma+\varepsilon_{i}$

Donde $R_{i}^{*}$ es una variable latente que denota la diferencia entre la utilidad de laborar para una IME, $U_{i}^{L}$, y la utilidad o beneficio de no laborar para una IME, $U_{i}^{N L}$. El individuo decidirá laborar en una IME si $R_{i}^{*}=U_{i}^{L}-U_{i}^{N L}>0$. El término $X_{i}^{\prime} \gamma$ provee un estimador de la diferencia en la utilidad de laborar 
para la IME $\left(U_{i}^{L}-U_{i}^{N L}\right)$ empleando el perfil de los individuos y las características de sus hogares $X_{i}$, como variables explicatorias, mientras que $\mathcal{E}_{i}$ es el término de error. Para estimar las ecuaciones (1) y (2), es necesario describir la relación que existe entre ser trabajador de una IME y su nivel de ingreso, ecuaciones que podrían ser interdependientes. Esto es, ser trabajador de una IME podría ayudar a incrementar el ingreso del hogar y contribuir a cambiar o no la condición de pobreza (de hogar pobre a no pobre); sin embargo, los hogares no pobres probablemente tendrán un perfil más ad-hoc para laborar para una IME. De este modo, la asignación de "tratamiento (individuo que trabaja para una IME)" no es aleatoria; dentro del grupo de los individuos que trabajan para una IME será sistemáticamente diferente. En especial el sesgo en la auto-elección ocurre si los factores inobservables influyen ambos términos de error en la ecuación de ingreso $\left(u_{i}\right)$, y en la decisión de laborar o no laborar $\left(\varepsilon_{i}\right)$, pudiendo resultar correlación en los términos de perturbación en las ecuaciones de ingreso (1) y decisión (2). De ahí, estimar la ecuación (1) a través de Mínimos Cuadrados Ordinarios (MCO) no es correcto porque se obtendrían estimadores sesgados e ineficientes.

Algunos autores han empleado el método de dos etapas de Heckman para corregir el sesgo, cuando la correlación de los dos términos de error es mayor a cero. Sin embargo, el enfoque depende de asumir el supuesto restrictivo de la distribución normal de los errores. Otro mecanismo para controlar el sesgo de la autoselección es utilizando variables instrumentales (vI); sin embargo, la mayor limitante de este método es la dificultad para encontrar e identificar VI en la estimación. Además, ambos métodos MCO y VI suelen tender a imponer una forma funcional lineal, donde el supuesto implica que los coeficientes sobre las variables de control de los individuos que laboran y los que no laboran en la IME son similares, por lo que este supuesto probablemente no corrige el sesgo, dado que los coeficientes pueden diferir (Jalan y Ravallion, 2003). Diferente a los métodos paramétricos anteriormente mencionados, es el método Propensity Score-Matching (PSM) pues no necesita supuestos acerca de la forma funcional para modelar una relación específica entre los resultados y la predicción de los resultados. El inconveniente del método PSM es el Supuesto de Independencia Condicional (CIA, por sus siglas en inglés), el cual expone que para un conjunto dado de covariables, la participación es independiente de los resultados potenciales. Smith y Todd (2005) argumentan que puede haber diferencias sistemáticas entre los que laboran y los que no laboran en una IME, incluso condicionando sobre variables observables. Jalan y Ravallion (2003) argumentan que los supuestos de selección sobre variables observables no son más restrictivos que aquéllos 
en los que se basan en método de dos-etapas de Heckman o vi cuando son utilizados en el análisis de datos de sección cruzada.

\section{Evaluación del problema y el método Propensity Score-Matching}

Una cuestión importante en la evaluación de impactos en el ingreso de los individuos que laboran y los que no laboran para una IME es la especificación del efecto promedio del "tratamiento" (Average Treatment Effect, ATE, por sus siglas en inglés). Rosenbaum y Rubin (1983) definen el ATE $\left(\Delta_{i}\right)$ en un marco contrafactual como:

$\Delta_{i}=Y_{i}^{L}-Y_{i}^{N L}$

Donde $Y_{i}^{L}$ y $Y_{i}^{N L}$ denotan el ingreso de un individuo $i$ que labora en una IME y el individuo que no labora en una IME, respectivamente. Para estimar el impacto de la ecuación (3), el problema se origina debido a que para un individuo normalmente sólo conocemos $Y_{i}^{L}$ o $Y_{i}^{N L}$, pero no para ambos individuos. Lo que normalmente se observa se puede expresar como:

$Y_{i}=D_{i} Y_{i}^{L}+\left(1-D_{i}\right) Y_{i}^{N L} \quad D_{i}=0,1$.

Denotando $P$ como la probabilidad de observar a un individuo con $D=1$, el efecto promedio del tratamiento (ATE), $\tau$, se puede especificar como:

$\tau=P \cdot\left[E\left(Y^{L} \mid D=1\right)-E\left(Y^{N L} \mid D=1\right]\right.$

$+(1-P) \cdot\left[E\left(Y^{L} \mid D=0\right)-E\left(Y^{N L} \mid D=0\right)\right]$

La ecuación (5) implica que el efecto de laborar en una IME para toda la muestra es el peso promedio del efecto de laborar de todos los empleados en la IME (tratamientos) y los que no laboran (controles), con cada peso por su relativa frecuencia. El principal problema de la inferencia causal proviene del

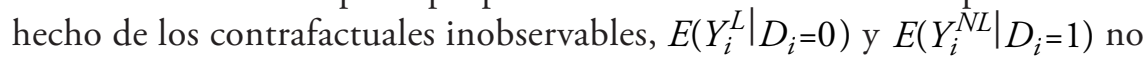
se pueden estimar (Smith y Todd, 2005).

Particularmente, cuando la disponibilidad de datos no provee información sobre la situación contrafactual, el problema de información ausente aumenta, la cual se requiere para estimar directamente los efectos en el ingreso por trabajar en una IME. Este estudio aborda este problema usando el método Propensity Score-Matching (PSM) que resume las características de 
los pre-tratamientos para cada objetivo dentro de una variable índice, luego utiliza el Propensity Score para ser aparejado o Matching con individuos lo más semejantes posible (Rosenbaum y Rubin, 1983). El PSM, que es la probabilidad de ser asignado a tratamiento condicionado sobre variables de pre-tratamiento, está dado por:

$$
p(X)=\operatorname{Pr}[D=1 \mid X]=E[D \mid X] ; p(X)=F\left\{h\left(X_{i}\right)\right\}
$$

Donde $F\{\cdot\}$ representa una distribución acumulativa normal o logística y $X$ es un vector de características de pre-tratamientos. ${ }^{3}$

Para estimar los efectos del tratamiento con base en el método de PSM se requiere dos supuestos importantes. El primero es el Supuesto de Independencia Condicional (CIA) mencionado arriba. El segundo es el efecto promedio del tratamiento para los tratamientos (ATT, por sus siglas en inglés), definido solamente dentro de la región de soporte común. Estos supuestos aseguran que los individuos con los mismos valores del vector de características $\mathrm{X}$ tienen una probabilidad positiva de ser participantes o no (Heckman et al., 1997).

Una vez que el Propensity Score es calculado, el ATT puede ser estimado como sigue:

$A T T=E\left\{Y_{i}^{L}-Y_{i}^{N L} \mid D_{i}=1\right\}$,

$A T T=E\left[E\left\{Y_{i}^{L}-Y_{i}^{N L} \mid D_{i}=1, p(X)\right\}\right]$,

$A T T=E\left[E\left\{Y_{i}^{L} \mid D_{i}=1, p(X)\right\}-E\left\{Y_{i}^{N L} \mid D_{i}=0, p(X)\right\} \mid D_{i}=1\right]$

Diferentes métodos se han propuesto en la literatura para aparejar similares o muy semejantes individuos que laboran y no laboran en una IME. El método comúnmente usado es "el vecino más cercano" (NNM, por sus siglas en inglés) y el Kernel-based (квм, por sus siglas en inglés). El método NNM consiste en aparejar a cada individuo con tratamiento con el individuo control que tiene el Propensity Score más cercano entre el tratamiento y el control, o dicho de

3 El método PSM controla la "auto-selección" mediante la creación de un "contrafactual” para el grupo de individuos que laboran en una IME. El PSM construye un grupo de comparación estadística mediante el grupo de individuos que laboran y los individuos que no laboran en una maquila. Así entonces, el PSM intenta construir una base de información tipo experimental, condicionada sobre características observables, resultando el proceso de selección aleatorio. 
otra forma, el individuo lo más similar posible que labora con el que no labora en la IME. Normalmente se aplica este método con reemplazos. El segundo paso es calcular las diferencias, para nuestro caso ingreso total anual, para cada "par" de unidades aparejadas, y finalmente el ATT se obtiene como el promedio de todas las diferencias.

\section{INFORMACIÓN Y FUENTES}

Los datos y la información que se utilizaron en este estudio, como se ha dicho en la introducción, son parte de un proyecto de investigación más amplio, Programa Dinámicas Territoriales Rurales (DTR) Yucatán, ${ }^{4}$ que consistió en tres etapas 1A, 2A y 2B. La primera etapa, elaborada por Yúnez et al. (2009), consistió en el análisis de una tipología de territorios rurales basada en mejoras o detrimentos a nivel de bienestar en el plano municipal ${ }^{5}$ nacional para el periodo 1990-2005. La totalidad de municipios que conforman la República Mexicana fueron analizados, es decir, 2,454 municipios. En la medición de los niveles de bienestar se estimaron tres indicadores: consumo per cápita, incidencia de la pobreza y coeficientes de desigualdad (Gini). Los resultados ponen de manifiesto la elevada heterogeneidad en los patrones de bienestar existentes en el país; no obstante, hay determinadas áreas en las cuales se sugiere haberse gestado condiciones de desarrollo económico regional. Los municipios contiguos con tipología similar se pueden identificar con un patrón de desarrollo común. De acuerdo con los resultados de Yúnez et al. (2009), es reducido el número de municipios que ha experimentado mejoras significativas en los tres indicadores de bienestar durante el periodo de estudio 1990-2005. El territorio СНAн propuesto aquí para su estudio, tiene esta tipología, es decir, con mejoras en los tres indicadores de bienestar.

La información fue recabada a partir de un cuestionario aplicado a una muestra representativa de los hogares del territorio снAн en la etapa 2B. Y a su vez, la selección de la muestra partió del listado de viviendas que se elaboró en la etapa 2A a partir de datos del Conteo de Población y Vivienda 2005 del Instituto Nacional de Estadística y Geografía (INEGI). Para asegurar la representatividad

4 Para indagar más al respecto, se invita al lector visitar el sitio en la red de RIMISP, Centro Latinoamericano para el Desarrollo Rural, programa DTR.

5 En México, el municipio es el nivel de división político-administrativo más bajo. 
de los más pobres se agrupó a los hogares de CHAH en dos estratos de marginación: alto (2,839 hogares) y medio (812 hogares) de acuerdo con lo que establece el Consejo Nacional de Población (CONAPO). A partir del diseño muestral - probabilístico y estratificado- se seleccionaron 251 hogares para encuestar: 186 del estrato alto y 65 del medio. Es muy importante aclarar que se excluyeron los hogares de la cabecera municipal de Acanceh por ser una localidad de baja marginación, por no ser rural y por limitaciones logísticas y financieras.

\section{Características generales de CHAH}

El territorio en estudio está conformado por cuatro municipios: Cuzamá, Homún, Acanceh y Huhí ( $\mathrm{CHAH})$, que en conjunto comprenden un poco más de 688 kilómetros cuadrados, localizado en la antigua región henequenera de Yucatán, y caracterizado como una de las zonas con las mayores concentraciones poblacionales y maya-hablantes de la entidad. La población que habita en el territorio CHAH es de aproximadamente 32,401 habitantes de acuerdo al Censo de Población y Vivienda 2010 (INEGI, 2011) con una distancia promedio de 33 a 52 kilómetros de la capital estatal, Mérida, según la ruta que se tome.

El principal motor de la economía en el territorio son las maquiladoras, entre las que destacan: Manufacturera Lee de México s. A. de c. v. en Acanceh, de capital extranjero orientada al mercado internacional, que emplea a 1,579 personas (el 73\% son del territorio) (Yúnez et al., 2011); también destaca la industria pecuaria para la producción de carne de ave, cerdo y bovino. Otro sector importante, pero de producción al nivel de hogar, es la manufactura y confección de la ropa típica yucateca y la manufactura de artesanías locales (hipiles y hamacas); y con menor importancia, la agricultura tradicional o milpa ${ }^{6}$ destinada prácticamente al autoconsumo. Finalmente, el trabajo no agrícola dentro y fuera del territorio es un componente importante del ingreso de los hogares, siendo los principales sitios de demanda de trabajo no agrícola: Mérida, Acanceh, Valladolid y Cancún (este último ubicado en el estado vecino de Quintana-Roo).

Ahora bien, para definir el territorio CHAH como tal, hay investigaciones previas en las que se indagó si los cuatro municipios contiguos: Cuzamá, Homún, Acanceh y Huhí, que tienen dinámicas positivas en materia de bienestar, forman

6 La milpa es el cultivo agrícola tradicional que comprende la asociación de maíz, frijol y calabaza, con variantes entre ibes, espelón u otros tipos de frijoles. La actividad es intensiva en mano de obra y se emplea el método milenario de roza, tumba y quema. 
un territorio. Yúnez et al. (2009) y Paredes et al. (2010) concluyen, de estudios a profundidad de carácter cualitativo y cuantitativo, que el CHAH es un territorio a partir de aplicar diferentes conceptos: nodalidad, homogeneidad, complementariedad, plan, así como la noción antropológica de identidad cultural, que son necesarios para definir un territorio.

\section{Cálculo de los perfiles de pobreza y desigualdad para el CHAH}

La estimación de los perfiles de pobreza alimentaria en CHAH (la cual se define como la proporción de hogares cuyo gasto ${ }^{7}$ por persona es menor al necesario para cubrir las necesidades de alimentación básicas) indican que ésta representó el $30 \%$ de sus hogares rurales en 2009 . El porcentaje es muy cercano con el prevaleciente en todo el México rural durante 2008: de 31.8\%; para el año 2009 este porcentaje debe ser ligeramente mayor si se toma en cuenta que la última creció debido a los efectos de la crisis internacional.

Ahora bien, para medir la severidad de la pobreza es necesario calcular los índices Foster, Greer y Thorbecke o FGT (1984), con los cuales es posible medir: la incidencia ( $\alpha_{0}=$ proporción de hogares pobres en la población en estudio), la profundidad o intensidad $\left(\alpha_{1}=\right.$ brecha promedio que existe entre los ingresos o gastos de los hogares en condición de pobreza y la línea de pobreza), y su severidad $\left(\alpha_{2}=\right.$ brecha proporcional entre los pobres). El Cuadro 1 muestra los resultados para la línea monetaria de pobreza alimentaria.

Para calcular las líneas monetarias de pobreza se estimó y actualizó el costo promedio de la canasta de consumo alimentaria y no alimentaria a partir de la metodología oficial para la medición de la pobreza en México, propuesta por el Comité Técnico para la Medición de la Pobreza (СтмP) en 2002, la cual define tres medidas alternativas de incidencia de pobreza: 1) pobreza alimentaria (línea 1), referida a la población cuyo ingreso per cápita mensual no es suficiente para adquirir una canasta básica alimentaria; 2) pobreza de capacidades (línea 2), o población cuyo ingreso per cápita mensual no es suficiente para adquirir la canasta básica alimentaria y, además, satisfacer los gastos necesarios en salud y educación; y 3) pobreza de patrimonio (línea 3), o población cuyo ingreso per

7 El gasto puede ser considerado un mejor indicador del nivel de satisfacción de las necesidades de los hogares, bajo el supuesto de que éstos tienen acceso a mecanismos que les permiten estabilizar su consumo en periodos en los cuales el ingreso presenta una alta inestabilidad (Yúnez et al., 2009). 
cápita mensual no es suficiente para satisfacer los gastos en alimentación, salud, educación y, además, los gastos necesarios en vivienda, vestuario y transporte.

Adicionalmente, para considerar la composición demográfica de los hogares en términos per cápita se ajustó el tamaño del hogar mediante escalas de adulto equivalente. El tamaño del hogar en unidades de adulto equivalente se construye usando distintas ponderaciones para los grupos de edad y sexo, de acuerdo con la definición proporcionada por el Instituto Nacional de Nutrición (Skoufias, 2005). Finalmente la actualización de los precios de la canasta alimentaria y no alimentaria, conforme al índice nacional de precios al consumidor (Banco de México, 2009) arrojó un costo mensual por persona para las zonas rurales, para la Línea 1 pobreza alimentaria MX\$756.55; Línea 2 pobreza de capacidades MX\$1,378.04; y Línea 3 pobreza de patrimonio MX $\$ 1,711.64$ todas a junio de 2010.

Cuadro 1. Índice FGT para línea 1 de pobreza alimentaria, 2009

\begin{tabular}{lc}
\multicolumn{1}{c}{ Indices } & СНAH (\%) \\
\hline$\alpha_{0}:$ Incidencia & 35.1 \\
$\alpha_{1}$ : Profundidad o intensidad & 10.7 \\
$\alpha_{2}:$ Severidad & 4.8 \\
Número de habitantes $(n)=1,101$ & 251 hogares \\
\hline
\end{tabular}

Fuente: Estimaciones propias con base en encuesta a hogares СНАН.

Asimismo, la desigualdad en el снан es baja ya que el coeficiente de $G i n i^{8}$ que se calculó para 2009 con datos de la encuesta mencionada es de 0.35 , mientras que, según cálculos del CONEVAL, el coeficiente de desigualdad para el estado de Yucatán fue de 0.49 en 2008 (CONEval, 2011).

No obstante que los perfiles de pobreza son robustos, y son una muestra fehaciente de la severidad y profundidad del fenómeno de los hogares del CHAH, con el fin de abundar en el fenómeno, el Cuadro 2 reporta la relación que existe en la diferencia promedio de edad, educación del jefe del hogar, quiénes típicamente toman las decisiones en el hogar y las tres líneas de pobreza. La evidencia

8 El cálculo del Gini se efectuó con el módulo de STATA “ “fastgini” Sajai (2007). 
real muestra que los hogares no pobres son encabezados por individuos más jóvenes, paralelamente comandados por jefes de hogar con mayor nivel de educación formal.

Cuadro 2. Diferencia en promedios de edad y educación de los jefes del hogar, según nivel de pobreza, 2009

\begin{tabular}{lrrrrrrr}
\hline & \multicolumn{3}{c}{ Línea pobreza 1} & \multicolumn{2}{c}{ Línea pobreza 2 } & \multicolumn{2}{c}{ Línea pobreza 3 } \\
\cline { 2 - 8 } & \multicolumn{1}{c}{ Pobre } & No pobre & Pobre & No pobre & Pobre & No pobre \\
\hline Edad promedio jefe del hogar & 47.9 & 46.6 & 47.8 & 44.9 & $47.9^{\star}$ & $43.4^{\star}$ \\
Educación promedio jefe del hogar & 4.6 & 5.1 & $4.7^{\star}$ & $5.8^{*}$ & $4.7^{\star \star}$ & $6.3^{\star \star}$ \\
\hline
\end{tabular}

$n=247$ hogares; prueba $t$, diferencia en promedios: * $10 \% ;{ }^{\star \star} 5 \% ;{ }^{\star \star \star} 1 \%$ de significancia.

Fuente: Estimaciones propias con base en encuesta a hogares СНАH.

\section{Estructura socioeconómica de los hogares del CHAH}

Esta sección muestra la estructura socioeconómica del CHAH, con énfasis en las características de los individuos mayores a 18 años, ${ }^{9}$ es decir, 755 habitantes que son el núcleo de análisis para evaluar el impacto económico derivado del establecimiento de la IME en el territorio en estudio. El Cuadro 3 reporta una estadística básica de los factores empleados en la evaluación de impactos. Destaca la edad de los habitantes mayores a 18 años con un promedio de edad de 39 años, la educación promedio de 5.6 años; el 57\% de los entrevistados reportó tener buena salud y el 29\% declaró laborar en su localidad de origen.

El tamaño de la familia promedio, ajustado por adulto equivalente, es de aproximadamente 4.45 miembros por hogar, y los detalles se presentan en el Cuadro 4. Es de importancia resaltar que en promedio $26 \%$ de los ingresos de los hogares proviene de programas de política pública, ya sea de tipo federal o estatal. El 23\% de los hogares encuestados reportó tener algún tipo de actividad comercial o de servicios. Mientras que 20\% declaró tener milpa, básicamente para el autoconsumo familiar. El 92\% de los hogares entrevistados aseguró que la vivienda que habitan es propia.

9 En México, los ciudadanos mayores de 18 años adquieren la mayoría de edad para votar y trabajar. 
Cuadro 3. Descripción del perfil de los habitantes mayores a 18 años del CHAH, 2009

\begin{tabular}{llcc}
\hline \multicolumn{1}{c}{ Variable } & \multicolumn{1}{c}{ Descripción } & Media & $\begin{array}{c}\text { Desviación } \\
\text { Estándar }\end{array}$ \\
\hline Sexo & 1 = Si el miembro del hogar es hombre & 0.51 & 0.50 \\
Edad & Edad cumplida en años & 38.86 & 16.42 \\
Educación & Educación formal en años & 5.64 & 3.75 \\
Salud & 1 = Si el miembro reportó tener buena salud & 0.57 & 0.49 \\
Ocupación Local & 1 = Si el miembro se emplea en la localidad & 0.29 & 0.45 \\
\hline
\end{tabular}

$\mathrm{n}=755$ habitantes mayores a 18 años de edad.

Fuente: Estimaciones propias con base en encuesta a hogares СНAH.

Cuadro 4. Descripción de las características de los hogares del CHAH, 2009

\begin{tabular}{|c|c|c|c|}
\hline Variable & Descripción & Media & $\begin{array}{l}\text { Desviación } \\
\text { Estándar }\end{array}$ \\
\hline Familia & $\begin{array}{l}\text { Número de miembros del hogar en adulto } \\
\text { equivalente }\end{array}$ & 4.45 & 1.96 \\
\hline Procampo & 1 = Si el hogar recibió subsidio de Procampo & 0.06 & 0.23 \\
\hline S. Popular & 1 = Si el hogar recibió Seguro Popular & 0.20 & 0.40 \\
\hline 70 y más & $\begin{array}{l}1 \text { = Si el hogar recibió subsidio del programa } \\
70 \text { y más }\end{array}$ & 0.09 & 0.28 \\
\hline Oportunidades & 1 = Si el hogar recibió subsidio de Oportunidades & 0.47 & 0.50 \\
\hline Porcentaje ingresos & Porcentaje del ingreso vía subsidios & 26.15 & 38.31 \\
\hline Vivienda propia & 1 = Si la vivienda es propia & 0.92 & 0.27 \\
\hline Sólo gas & I = Si el hogar cocina únicamente con gas LP & 0.09 & 0.28 \\
\hline Comercio y servicios & $\begin{array}{l}1 \text { = Si el hogar reportó tener comercio } 0 \\
\text { servicio vigente }\end{array}$ & 0.23 & 0.42 \\
\hline Ganado & 1 = Si el hogar reportó tener ganado mayor & 0.08 & 0.27 \\
\hline Milpa & 1 = Si el hogar reportó tener milpa & 0.20 & 0.40 \\
\hline Crédito formal & 1 = Si el hogar reportó tener crédito familiar & 0.18 & 0.39 \\
\hline
\end{tabular}

$\mathrm{n}=247$ hogares.

Fuente: Estimaciones propias con base en encuesta a hogares СНАH. 
Dentro de esta estadística descriptiva básica, se elaboró un análisis somero de la diferencia en los promedios, prueba $t$, para los individuos que dijeron laborar para una IME y los que declararon laborar en cualquier otro tipo de actividad; los resultados se reportan en el Cuadro 5. El primer hallazgo de relevancia es la diferencia que existe en el ingreso total anual entre los individuos que laboran en una IME y los que laboran en cualquier otro sector, siendo la diferencia estadísticamente significativa. En segundo plano, destaca la diferencia significativa para la edad y los años de educación formal de los individuos que declararon laborar para una IME; los hallazgos que coinciden con lo reportado por Castilla y Torres (2009) argumentan que los empresarios de la IME prefieren emplear jóvenes, con primera inserción al trabajo laboral, sin experiencia fabril y con estudios de secundaria. En este mismo sentido, de acuerdo con Gómez (2004) desde 1995 ha aumentado a nivel nacional el peso del personal más calificado (personal técnico de producción y empleados administrativos), perdiendo peso el personal obrero. Por otro lado, la mayoría de los individuos (72\%) que laboran en la maquila reportaron tener buena salud, mientras que el 23\% declaró laborar en la localidad; para ambos casos las diferencias son significativas.

Cuadro 5. Diferencia en las características de los individuos que Laboran y No Laboran en una maquiladora de exportación (media simple)

\begin{tabular}{lccc}
\hline \multicolumn{1}{c}{ Descripción } & $\begin{array}{c}\text { Labora para una } \\
\text { IME }\end{array}$ & $\begin{array}{c}\text { No Labora en } \\
\text { la IME }\end{array}$ & Valor - $\boldsymbol{~}$ \\
\hline Ingreso completo anual & $\$ 28,282.0$ & $\$ 13,611.2$ & -3.58 \\
\hline I = Si el miembro del hogar es hombre & 0.92 & 0.49 & -5.36 \\
Edad cumplida en años & 29.10 & 40.21 & 4.21 \\
Educación formal en años & 8.08 & 5.38 & -4.47 \\
l = Si el miembro reportó tener buena salud & 0.72 & 0.57 & -1.84 \\
I = Si el miembro se emplea en la localidad & 0.23 & 0.29 & -1.84 \\
\hline $\mathrm{n}=$ 729 habitantes mayores a 18 años de edad. & & \\
Fuente: Estimaciones propias con base en encuesta a hogares CHAH. & &
\end{tabular}

Finalmente, el Cuadro 6 muestra las características de los hogares donde viven los individuos que reportaron laborar en una maquiladora. Existiendo una diferencia estadísticamente significativa en el subsidio del Seguro Popular, el resultado podría sugerir que los individuos que se emplean en cualquier otro 
tipo de trabajo no tienen prestaciones sociales o un contrato laboral específico; razón de ello es la diferencia estadísticamente significativa en el $20 \%$ de hogares que reciben el subsidio y que no laboran en una maquila. Respecto al acceso a un crédito formal de alguna institución bancaria o caja popular establecida en el territorio, el 30\% de los hogares con miembros empleados en el sector maquilador reportaron tener algún tipo de crédito formal; la diferencia estadísticamente significativa podría sugerir algún tipo de seguridad para contratar el crédito, pues en muchos de los casos las compañías crediticias solicitan solvencia económica o comprobantes de nómina para otorgar el crédito. Sin lugar a dudas, tener un empleo asalariado reduce la vulnerabilidad, otorgando en cierta forma estabilidad en el bienestar y la posibilidad de tener una línea de crédito para cubrir eventualidades o la usual compra de artículos para el hogar a plazos.

Cuadro 6. Diferencia en las características de los hogares según empleados o no empleados en maquiladoras (media simple)

\begin{tabular}{lccc}
\hline \multicolumn{1}{c}{ Descripción } & $\begin{array}{c}\text { Labora para } \\
\text { Una IME }\end{array}$ & $\begin{array}{c}\text { No Labora en } \\
\text { la IME }\end{array}$ & Valor - $\dagger$ \\
\hline Número de miembros del hogar en adulto equivalente & 4.57 & 4.45 & -0.25 \\
1 = Si el hogar recibió subsidio de Procampo & 0.03 & 0.08 & 1.21 \\
I = Si el hogar recibió Seguro Popular & 0.08 & 0.21 & 2.01 \\
I = Si el hogar recibió el programa 70 y más & 0.03 & 0.09 & 1.43 \\
1 = Si el hogar recibió subsidio de Oportunidades & 0.53 & 0.50 & -0.48 \\
Porcentaje del ingreso vía subsidios & 16.90 & 26.16 & 1.44 \\
1 = Si la vivienda es propia & 0.95 & 0.94 & -0.24 \\
I = Si el hogar cocina únicamente con gas LP & 0.15 & 0.08 & -1.63 \\
I = Si el hogar reportó tener algún comercio 0 servicio & 0.18 & 0.27 & 1.24 \\
vigente & & & \\
1 = Si el hogar reportó tener ganado mayor & 0.08 & 0.07 & -0.05 \\
1 = Si el hogar reportó tener milpa & 0.26 & 0.24 & -0.31 \\
I = Si el hogar reportó tener crédito familiar & 0.31 & 0.16 & -2.45 \\
\hline
\end{tabular}

$\mathrm{n}=247$ hogares.

Fuente: Estimaciones propias con base en encuesta a hogares СНАН. 
Por último, existe otra diferencia relevante entre los hogares con miembros empleados en la industria maquiladora y los que no se emplean allí, respecto a cocinar únicamente con gas, aunque la diferencia no es estadísticamente significativa, o mayor al 10\%. En el medio rural mexicano, el hecho de cocinar con gas reduce muchas vulnerabilidades al interior del hogar y contribuye al bienestar y la salud de sus habitantes, además de mayor disponibilidad de tiempo para otras labores domésticas. Está comprobado que la recolección de leña y a su vez cocinar con biomasa demanda tiempo en su recolección y aumenta el número de enfermedades crónicas respiratorias, entre otros impactos ambientales. La evidencia empírica demuestra que cocinar con gas acarrea un mayor nivel de bienestar que con el sustituto energético: leña (INE, 2005; Troncoso et al., 2007).

\section{ANÁLISIS DE IMPACTOS}

En el instrumento de análisis se mencionó que el primer paso en la evaluación de impactos es calcular el Propensity Score o parámetros de un modelo de regresión dicotómico, en este caso, un modelo logístico o logit. El Cuadro 7 reporta los parámetros de los factores socioeconómicos, el perfil de los individuos y las características de sus hogares, que explican la probabilidad de laborar para la industria maquiladora de exportación. La mayoría de las variables arrojaron el signo esperado conforme a la teoría económica y son altamente significativas, lo cual muestra que el modelo es robusto. Una vez calculado el Propensity Score, se procedió a realizar la evaluación de impactos a través de dos algoritmos NNM y квм para efectuar el Matching o aparejamiento de los individuos que declararon laborar (tratamientos) con los que no laboraron para una IME (controles). Esto se reporta en el Cuadro 8.

El primer conjunto de variables que versa sobre el perfil de los individuos, es altamente significativo y con el signo esperado: Sexo (sex), Edad (age) y Educación $(e d u)$ que se puede corroborar con la teoría económica y lo reportado en la literatura; por ejemplo, el coeficiente de la variable educación tiene el signo esperado y es estadísticamente significativo, coincidiendo con los argumentos de Yúnez et al. (2011) de la transformación económica del territorio caracterizada por el cambio en el peso relativo de las distintas fuentes de ingreso de sus habitantes y por una diferenciación demográfica en las ocupaciones de su fuerza de trabajo, explicada en gran parte por la mayor escolaridad de los jóvenes. Por otro lado, el coeficiente para la edad resulta con el signo esperado y coincide con los hallazgos de Castilla y Torres (2009), para quienes la mayoría de las maquilas prefieren contratar a hombres y mujeres jóvenes. 
Cuadro 7. Parámetros modelo Logit, sobre los factores que influyen para ser empleado en una maquila (Propensity Score)

\begin{tabular}{lccc}
\hline \multicolumn{1}{c}{ Variable } & Coeficiente & Error Estándar & Valor-z \\
\hline Sex & 5.901 & 1.579 & 3.74 \\
Age & -0.117 & 0.035 & -3.34 \\
Edu & 0.115 & 0.052 & 2.19 \\
Aefsize & -9.447 & 4.855 & -1.95 \\
Godhealt & 0.682 & 0.441 & 1.55 \\
Ocuplocal & 0.582 & 0.490 & 1.19 \\
Procampo & -1.176 & 1.121 & -1.05 \\
Spopu & -1.002 & 0.646 & -1.55 \\
Más & -0.451 & 1.449 & -0.31 \\
Oportuni & 0.536 & 0.418 & 1.28 \\
Shareppp & -0.007 & 0.006 & -1.14 \\
Vivprop & 1.240 & 0.991 & 1.25 \\
Onlygas & 1.315 & 0.595 & 2.21 \\
Comserv & -0.830 & 0.462 & -1.80 \\
Ganado & 0.273 & 0.717 & 0.38 \\
Milpa & 0.602 & 0.495 & 1.22 \\
Creditof & 0.921 & 0.430 & 2.14 \\
C & 4.440 & 5.411 & 0.82 \\
\hline
\end{tabular}

$\mathrm{n}=730$ habitantes; Prob. $>$ Chi2 $(17)=0.0000 ;$ Pseudo R2 $=0.2809$.

Fuente: Elaboración propia con resultados de STATA.

Otra variable significativa, y que coincide con la teoría económica, es el tamaño del hogar (aefsize): a mayor número de miembros en la familia se reduce la probabilidad de emplearse en un trabajo asalariado, pues mayor es el capital humano y mano de obra familiar para realizar actividades.

Aunque no sea significativo, el signo positivo del coeficiente del programa gubernamental federal Oportunidades (oportuni) concuerda con los hallazgos de Yúnez et al. (2011), donde el aumento en la escolaridad de niños y jóvenes causado por el programa promueve el interés de éstos por trabajar en actividades no agropecuarias asalariadas. En la misma dirección se encuentran las variables con signos negativos y esperados para los programas de Procampo (procampo), 
Seguro Popular (spopu) y "Setenta y más" (más); todas ellas no estadísticamente significativas. En esta misma línea de argumentos, la variable de la proporción del ingreso del hogar proveniente de los programas de política pública social o productiva (shareppp) resulta con el signo esperado aunque no sea significativa y puede sugerir que a mayor ingreso reduce la probabilidad de laborar para una IME.

Cuadro 8. Efectos marginales de los factores que determinan el empleo en una maquiladora

\begin{tabular}{lccc}
\hline \multicolumn{1}{c}{ Variable } & $d y / d x$ & Error Estándar & \multicolumn{1}{c}{ Valor-z } \\
\hline Sex & 0.061 & 0.021 & 2.92 \\
Age & -0.001 & 0.000 & -2.61 \\
Edu & 0.001 & 0.001 & 1.70 \\
Aefsize & -0.098 & 0.055 & -1.79 \\
Godhealt & 0.007 & 0.005 & 1.49 \\
Ocuplocal & 0.006 & 0.005 & 1.14 \\
Procampo & -0.012 & 0.012 & -1.00 \\
Spopu & -0.010 & 0.007 & -1.53 \\
Más & -0.005 & 0.014 & -0.33 \\
Oportuni & 0.006 & 0.004 & 1.27 \\
Shareppp & 0.000 & 0.000 & -1.09 \\
Vivprop & 0.013 & 0.011 & 1.13 \\
Onlygas & 0.014 & 0.007 & 1.96 \\
Comserv & -0.009 & 0.005 & -1.64 \\
Ganado & 0.003 & 0.007 & 0.39 \\
Milpa & 0.006 & 0.005 & 1.23 \\
Creditof & 0.010 & 0.005 & 1.82 \\
\hline
\end{tabular}

Fuente: Elaboración propia con resultados de STATA.

El coeficiente positivo, aunque no significativo de ocupación en la localidad (ocuplocal) coincide con los trabajos elaborados por Castilla y Torres (2009) con estudios en varias zonas del agro yucateco, al mencionar que el despliegue rural de la IME constituye una oportunidad sistemática para que las mujeres de las localidades rurales puedan integrarse al mercado de trabajo sin tener que separarse 
radicalmente del hogar. En este mismo sentido, Yúnez et al. (2011) afirman que la poca emigración en el CHAH no es común en el México rural, indicando que en el territorio en cuestión y su región hay fuentes alternativas de ingreso en comparación a las existentes en otros mercados de trabajo en el México urbano o en los EUA.

Con relación a cocinar únicamente con gas (Onlygas), el signo positivo y significativo indica que los hogares con mayor bienestar pueden ser causa-efecto de laborar en la IME. Este resultado puede fortalecerse con el obtenido por Ortiz (2009) que muestra que en Yucatán el uso de gas está relacionado con mejores condiciones de vida derivadas de dedicarse a sectores diferentes al primario, o por trabajar en actividades mejor remuneradas.

La variable Comercio y Servicios (comserv) resulta con el signo esperado y estadísticamente significativo, lo que sugiere que si el hogar y sus miembros tienen alguna actividad económica orientada hacia el comercio (tienda, farmacia, por ejemplo) o prestación de servicios (tricitaxis, taxis foráneos, por ejemplo), se reduce la probabilidad de laborar para una maquiladora.

\section{Evaluación de impactos en el ingreso de los individuos}

Una vez efectuado el Propensity Score o modelo de regresión dicotómico mediante la regresión logística, se procede al análisis no factorial empleando los algoritmos NNм у квм, cuyos resultados se reportan en el Cuadro 9.

Cuadro 9. Promedio de los efectos del tratamiento sobre el ingreso completo anual

\begin{tabular}{lcccc}
\hline Algoritmo Matching & Variable resultado & ATT & $\begin{array}{r}\text { Número } \\
\text { Tratamientos }\end{array}$ & $\begin{array}{c}\text { Número de } \\
\text { Controles }\end{array}$ \\
\hline Nearest Neighbor Matching & Ingreso completo anual & $\begin{array}{r}\$ 9,448.81 \\
(2.05)\end{array}$ & 39 & 691 \\
& & & & \\
Kernel-based Matching & Ingreso completo anual & $\begin{array}{r}\$ 8,419.08 \\
(2.38)\end{array}$ & 37 & 691 \\
& & & & \\
\hline
\end{tabular}

Fuente: Elaboración propia con resultados de STATA.

El impacto en el ingreso completo anual de habitantes que laboran para la IME establecida en el territorio $\mathrm{CHAH}$, es una prueba de los beneficios que deja 
la industria en el ingreso directo de los habitantes. Existe pues, una clara diferencia que va de $\mathrm{MX} \$ 8,419.08$ a MX\$9,448.81 pesos, a diferencia de laborar en cualquier otro sector.

Los hallazgos aquí reportados coinciden con la evidencia empírica de Castilla y Torres (2009): la incorporación de mujeres rurales e indígenas mayas al mercado laboral, que generalmente habitan en zonas asoladas por la pobreza y carentes de fuentes de empleo a causa de la larga y profunda crisis del agro. El empleo asalariado en estas maquiladoras les permite aguinaldo, pago de vacaciones, utilidades, el acceso a la seguridad social para su familia, prestaciones y otros beneficios (bonificaciones por productividad, asistencia y puntualidad) que no pueden acceder en otras fuentes, en su mayoría no formales, de empleo dentro del territorio; lo que ayuda a la economía del hogar y de CHAH. Como también afirman Castilla y Torres (2010), "la IME contribuyó de forma importante con la creación de empleo formal, medianamente aceptable, en términos del trabajo decente de la Organización Internacional del Trabajo".

La derrama económica es clara, pues los habitantes que laboran para una IME tienen un ingreso mensual en promedio de MX\$744 pesos más que los que trabajan en otras actividades distintas a las maquiladoras; además este ingreso es neto, ya que laboran en la localidad o muy próximos a su lugar de origen o residencia, ahorrándose así los gastos de desplazamiento (Albornoz et al., 2000). Otra ventaja al laborar en maquiladora es que una parte de los ingresos obtenidos por los trabajadores son gastados en bienes y servicios dentro de la localidad, provocando un efecto multiplicador en la economía de СHAH.

Así concluimos que los que laboran en una IME proporcionan una fuente de ingresos importante para sus familias y para el territorio. Este recurso monetario es neto, que junto con las prestaciones hacen que parte de la población se decida a laborar en esta actividad productiva, de modo que puede ayudar a las condiciones económicas precarias de los hogares de la antigua zona henequenera.

\section{DISCUSIÓN}

Los hallazgos en este estudio econométrico sobre los efectos de la industria maquiladora de exportación en el ingreso directo de los habitantes en cuatro municipios de la antigua región henequenera, particularmente en el territorio CHAH, esclarecen la importancia y relevancia del sector maquilador para cientos de hogares rurales de Yucatán. Los resultados del modelo logístico con sus signos esperados, ya sean positivos o negativos, su robustez y significancia nos muestran por qué los habitantes que laboran para las maquilas se deciden por 
esta actividad. Muestran también el beneficio que deja la derrama económica en el territorio СНAH. El artículo cumple con su objetivo de investigación y académico al valorar el efecto de la industria en el ingreso de los individuos, aplicando adicionalmente un método no paramétrico de evaluación.

El análisis econométrico factorial reveló también el perfil de los individuos que laboran en la industria, empleando a jóvenes, calificados a nivel secundaria y con buen estado de salud, entre otros factores. Nuestros hallazgos sugieren el rumbo que están tomando los jóvenes yucatecos que viven y se desenvuelven en el medio rural, siendo las maquiladoras una fuente segura y oportuna de empleo. De acuerdo con Gómez (2004), el aumento en la calidad y productividad de los trabajadores de las maquilas no sólo generó en los últimos años un incremento en las retribuciones reales de estos trabajadores, sino también un aumento con respecto al conjunto de los trabajadores mexicanos. La retribución media en la maquila en 2002 fue de $125.1 \%$ de la remuneración media mexicana, cifra que alcanzaba $102 \%$ en el caso de los obreros y $402 \%$ en el caso de los empleados.

Sin lugar a dudas, nuestro análisis coincide y soporta el argumento de Gómez (2004), dado que de mantener o incrementar la actividad de la industria maquiladora en Yucatán, dinamizará la economía por medio de la derrama económica del pago de sueldos y salarios, y por medio del potencial de vinculación con la planta productiva local y podrá contribuir con efectos multiplicadores en la adquisición de insumos locales y venta de productos elaborados a menor costo, porque de acuerdo con Gómez (2004) una de las principales limitaciones de la maquila para actuar como motor o catalizador del desarrollo económico mexicano es su escasa vinculación con el resto de la estructura productiva local debido al elevado consumo de insumos importados, lo que reduce la capacidad de la maquila para actuar como motor del resto de ramas de la economía. Por lo tanto, cualquier estrategia de desarrollo productivo en México implicará aumentar el grado de conexión entre maquila y empresas proveedoras locales. Tres posibilidades de actuación: $i$ ) aumentar el consumo de insumos nacionales en todas las ramas en las que opera la maquila, $i$ i) potenciar la actividad de aquéllas que más insumos nacionales consumen, y iii) cambiar la composición de los consumidos.

Es necesario realizar los objetivos anteriores; de otra forma la estrategia de desarrollo no impactará a largo plazo, tal como argumenta Biles (2004). Las maquiladoras han tenido un impacto mixto en el estado de Yucatán, sobre todo en el crecimiento económico en las áreas rurales. Se sugiere aumentar la transferencia tecnológica y mejorar el capital humano (especializado) de modo que se logre aumentar la producción de la IME y esto provoque que los trabajadores 
tengan un incremento en sus salarios. Si no se logra lo anterior en el corto plazo, se corre el riesgo de emular el florecimiento de la actividad henequenera del siglo pasado, la cual fracasó por la confianza en un solo producto.

Así, los resultados de nuestro estudio económico cuantitativo pueden enviar señales a los tomadores de decisión del Ejecutivo federal o, a nivel local, el Ejecutivo estatal de mantener, o en el mejor de los casos hacer atractiva la economía yucateca al capital extranjero para el establecimiento de la industria manufacturera y maquiladora de exportación, con mayores cuotas a los insumos locales, cuotas de ventas al mercado local y con un riguroso análisis de impacto ambiental, de modo que conviertan el crecimiento económico generado por la IME en desarrollo económico sustentable dentro de la antigua región henequenera, en zonas rurales y en todo el estado de Yucatán.

\section{BIBLIOGRAFÍA}

Albornoz, L. y R. Ortiz, "La industria maquiladora como sostén de las comunidades rurales de Yucatán”, Comercio Exterior, vol. 50, núm. 5, México, Banco Nacional de Comercio Exterior, mayo de 2000, pp. 448-452.

Banco de México, Indice de precios. 2009, disponible en: <http://www.banxico. org. $\mathrm{mx} />$

Biles, J., "Export-oriented Industrialization and Regional Development: A Case Study of Maquiladora Production in Yucatán, México", Regional Studies, 2004, vol. 38, num. 5, pp. 519-534.

Carrillo, J. y A. Hualde, "Maquiladoras de tercera generación. El caso de Delphi-General Motors", Comercio Exterior, vol. 47, núm. 9, México, Banco Nacional de Comercio Exterior, septiembre de 1997, pp. 747-758.

Castilla, B. y B. Torres, "Del hogar a la fábrica: trabajadoras de las empresas trasnacionales en Yucatán, México”, traCe 55 (en línea), junio 2009, pp. 31-52, disponible en: <http://trace.revues.org/index796.html>

Castilla, B. y B. Torres, "Crisis sobre crisis en la industria maquiladora de exportación y sus consecuencias en la ocupación. El caso de Yucatán”, El Cotidiano, núm. 162, México, UAM-Azcapotzalco, julio-agosto de 2010, pp. 43-56.

Conapo, Consejo Nacional de Población. 2011, disponible en: <http://www. conapo.gob. $\mathrm{mx} />$.

CONEval, vista en el sitio red, disponible en: <http://www.coneval.gob.mx>.

Foster, J., T. Greer y E. Thorbecke, "A Class of Decomposable Poverty Measures”, Econometrica, vol. 52, num. 3, mayo 1984, pp. 761-766. 
García, M. F., R. A. Posthuma, T. Mumford, M. Quiñones, “The Five Dimensions of Pay Satisfaction in a Maquiladora Plant in México", Applied Psychology, vol. 58, num. 4, octubre 2009, pp. 509-519.

Gómez, M., "El desarrollo de la industria de la maquila en México", Revista Problemas del Desarrollo, vol. 35, núm. 138, México, IIE-UnAM, julio-septiembre 2004, pp. 57-83.

Heckman, J., H. Ichimura y P. Todd, "Matching as an Econometric Evaluation Estimator", Review of Economic Studies, vol. 65, num. 2, abril 1998, pp. 261-294.

INegi, Conteo de Población y Vivienda 2005, Instituto Nacional de Estadística y Geografía 2005, disponible en: < www.inegi.gob.mx/>

INegi, Censo de Población y Vivienda 2010, Instituto Nacional de Estadística y Geografía 2011, disponible en: < www.inegi.gob.mx/>

Instituto Nacional de Ecología, Evaluación de concentraciones microambientales de particulas suspendidas en hogares rurales de Michoacán y actividades que influyen la exposición personal, 31 de octubre de 2005, disponible en: < http://www.ine.gob.mx/descargas/calaire/inf_final_estufas_2005.pdf >. Jalan, J. y M. Ravallion, "Does Piped Water Reduce Diarrhea for Children in Rural India?”, Journal of Econometrics, vol. 112, num. 1, enero 2003, pp. 153-173.

Ortiz, R., Extracción y uso de leña como recurso natural renovable: un enfoque de simulación dinámica, Tijuana, Universidad Autónoma de Baja California, 2009, tesis de doctorado.

Paredes, L., A. Yúnez, J. Méndez y J. Becerril, “СНAH, territorio en Yucatán: identidad, políticas públicas para su desarrollo”, ponencia presentada en el Encuentro Anual de RIMIsP: Territorios Rurales en Movimiento, Bogotá Colombia, 16-18 de marzo de 2010.

Quintal, A., Política económica y desarrollo industrial en Yucatán: el caso de la industria manufacturera, Ediciones de la Universidad Autónoma de Yucatán, 2010, México.

Rosenbaum, P. y D. Rubin, "The Central Role of the Propensity Score in Observational Studies for Causal Effects", Biometrika, vol. 70, num. 1, abril de 1983, pp. 41-55.

Sajai, Z. Fastgini: Stata Module to Perform Inequality Measures, versión 1.0, febrero 2007.

Secretaría de Fomento Económico, Estudio de coyuntura de la industria manufacturera, maquiladora y de servicios de exportación de Yucatán, Mérida, México, Gobierno del Estado de Yucatán, primer semestre de 2009. 
Skoufias, E., "ProgresA and its Impacts on the Welfare of Rural Households in México", Research Report, num. 139, Washington, International Food Policy Research Institute, 2005.

Smith, J. y P. Todd, "Does Matching Overcome LaLonde's Critique of NonExperimental Estimators?", Journal of Econometrics, vol. 125, num. 1-2, marzo-abril 2005, pp. 305-353.

Sklair, Leslie, Asembling for Development, the Maquila Industry in México and the United States, San Diego, University of California, the Center for U.S.Mexican Studies, 1993.

Taylor, G., "The Abject Bodies of the Maquiladora Female Workers on a Globalized Border", Race, Gender and Class, vol. 17, num. 3-4, 2010, pp. 349363.

Trívez, F., A. Reyes y F. Alinga, "Análisis cuantitativo de la coyuntura de la industria maquiladora de exportación mexicana”, artículo presentado en el Coloquio Mexicano de Economía Matemática y Econometría, ChetumalMéxico, Universidad de Quintana Roo, 21-25 mayo de 2007.

Troncoso, K., A. Castillo, O. Masera y L. Merino, "Social Perceptions About a Technological Innovation for Fuelwood Cooking: Case Study in Rural México", Energy Policy, vol. 35, num. 5, mayo de 2007, pp. 2799-2810.

Veloz, A., "Mujeres purépechas en las maquiladoras de Tijuana: entre la flexibilidad y significación del trabajo", Frontera Norte, vol. 22, núm. 44, Tijuana-México, El Colegio de la Frontera Norte, julio-diciembre de 2010, pp. 211-236.

Warden, S., "Assessing Export Platforms: The Case of the Maquiladora Sector in México", CAER II Discusion Paper num. 78, Cambridge, MA, Center for International Development-Harvard University, septiembre de 2000, pp. 5-31.

Wilson, P., Exports and Local Development México's New Maquiladoras, United States, University of Texas Press, 1992.

Yúnez, A., J. Arellano y J. Méndez, "México: consumo, pobreza y desigualdad a nivel municipal 1990-2005", documento de trabajo, núm. 31, Santiago de Chile, Programa Dinámicas Territoriales Rurales, RIMIsp-Centro Latinoamericano para el Desarrollo Rural, 2009.

Yúnez, A., L. Paredes, J. Méndez, I. Estrada, A. España, V. Serrano y J. Becerril, "La dinámica de un territorio en Yucatán", documento de trabajo, núm. 71, Santiago de Chile, Programa Dinámicas Territoriales Rurales, RIMISP-Centro Latinoamericano para el Desarrollo Rural, 2011. 